\title{
A importância da atenção Fisioterapêutica nos cuidados paliativos em pacientes com câncer
}

The importance of Physiotherapeutic care in palliative care in cancer patients

La importancia del cuidado de la fisioterapia en los cuidados paliativos en pacientes con câncer

Lucas Gabriel de Araújo Marcião ORCID: https://orcid.org/0000-0002-2592-8644 Centro Universitário da Amazônia, Brasil

E-mail: lucasgabrielaraujomarciao90@gmail.com Vitória Natalia Fernandes de Sousa ORCID: https://orcid.org/0000-0002-8705-5754 Centro Universitário da Amazônia, Brasil E-mail: vitorianatalha@gmail.com Patrícia Emerich Lima

ORCID: https://orcid.org/0000-0003-3736-840X Centro Universitário da Amazônia, Brasil E-mail: patricia.27emerick@hotmail.com Larissa Helanny Costa Lima ORCID: https://orcid.org/0000-0001-6708-8818 Centro Universitário da Amazônia, Brasil E-mail: fisiolarissa18@gmail.com

Emmanuele Figueiredo Marcião ORCID: https://orcid.org/0000-0003-4011-8073 Faculdade Pitágoras de Santarém, Brasil E-mail:emmanuelemarciao@gmail.com Eliandrea Lima Castro Alves

ORCID: https://orcid.org/0000-0001-8232-177X Centro Universitário da Amazônia, Brasil E-mail: eliandreaca65@gmail.com

Jhennifer Valéria Mattos Lira ORCID: https://orcid.org/0000-0002-0671-7921

Centro Universitário da Amazônia, Brasil E-mail: jmattoslira@gmail.com

Leandro Ferreira de Moura ORCID: https://orcid.org/0000-0001-8772-2089 Centro Universitário Pitágoras de Fortaleza, Brasil E-mail: leandromour2@gmail.com

Marta Maiara Silva Olivetto ORCID: https://orcid.org/0000-0003-2180-2304

Centro Universitário da Amazônia, Brasil E-mail: jwmmaaraolivetto@gmail.com

Geremias da Silva

ORCID: https://orcid.org/0000-0002-3331-7907

Faculdade Pitágoras de Santarém, Brasil E-mail: geremias.stm.silva@gmail.com

Orivaldo Pablo Costa Ferreira

ORCID: https://orcid.org/0000-0001-8778-2890

Faculdade Pitágoras de Santarém, Brasil E-mail: pablodafie153@gmail.com

Adriele Galvão Rodrigues

ORCID: https://orcid.org/0000-0001-7487-7604

Centro Universitário da Amazônia, Brasil E-mail: adrielegalvao96@gmail.com

Cristiane Pereira Alves

ORCID: https://orcid.org/0000-0003-1169-8830 Centro Universitário da Amazônia, Brasil

E-mail: cristianepereira9881@gmail.com Jamile Bentes Viana

ORCID: https://orcid.org/0000-0003-1797-906X Centro Universitário da Amazônia, Brasil E-mail: milly.bentes04@gmail.com 


\title{
Resumo
}

Indiara de Alencar

ORCID: ttps://orcid.org/0000-0003-3204-3278 E-mail: Indiara.alencarstm@gmail.com Centro Universitário da Amazônia, Brasil

A neoplasia maligna que é definida como câncer é uma patologia com características da ampliação celular sem controle que desencadeia um acúmulo de células malignas. Os cuidados paliativos necessitam de uma intervenção na atenção aos pacientes em estágios iniciais, avançados ou terminais, estimulando um processo de amenização ou de controlar os sinais e sintomas. Nesse contexto, o papel do fisioterapeuta é atuar de forma complementar ao tratamento paliativo, com o objetivo de melhorar a recuperação do indivíduo, restaurando as funções de diferentes partes do corpo. O objetivo desse trabalho é discutir a importância da intervenção da fisioterapêutica em oncologia, com enfoque em cuidados paliativos. Refere-se de uma revisão narrativa de literatura. Para este artigo foram realizadas buscas manuais na plataforma Google Scholar e selecionados artigos relacionados à temática. A partir dos dados apresentados, o papel da intervenção fisioterapêutica está relacionado à reabilitação em todas as fases da doença, na qual a dor é a ocorrência mais pertinente causando um grande desconforto ao paciente. A fisioterapia é uma área importante no tratamento de pacientes em fase terminal, reabilitando a condição motora e, com isso, inserir esse indivíduo na retomada da independência funcional. Por fim, estudos comprovam que a fisioterapia tem um papel importante nos cuidados paliativos, entretanto, as definições de atenção a pacientes paliativos são necessárias serem propagadas entre os fisioterapeutas e pesquisas são necessárias para um bom aperfeiçoamento profissional.

Palavras-chave: Fisioterapia; Cuidados paliativos; Neoplasias.

\begin{abstract}
The malignant neoplasm that is defined as cancer is a pathology with characteristics of uncontrolled cell enlargement that triggers an accumulation of malignant cells. Palliative care requires intervention in the care of patients in early, advanced or terminal stages, stimulating a process of easing or controlling the signs and symptoms. In this context, the role of the physiotherapist is to act in a complementary way to palliative treatment, with the aim of improving the individual's recovery, restoring the functions of different parts of the body. The objective of this work is to discuss the importance of physical therapy intervention in oncology, with a focus on palliative care. Refers to a narrative literature review. For this article, manual searches were made on the Google Scholar platform and selected articles related to the theme. From the data presented, the role of physical therapy intervention is related to rehabilitation in all stages of the disease, in which pain is the most relevant occurrence causing great discomfort to the patient. Physiotherapy is an important area in the treatment of terminally ill patients, rehabilitating the motor condition and, therefore, inserting this individual in the resumption of functional independence. Finally, studies prove that physiotherapy has an important role in palliative care, however, definitions of care for palliative patients are necessary to be propagated among physiotherapists and research is necessary for a good professional improvement.
\end{abstract}

Keywords: Physical therapy specialty; Palliative care; Neoplasms.

\begin{abstract}
Resumen
La neoplasia maligna que se define como cáncer es una patología con características de agrandamiento celular descontrolado que desencadena una acumulación de células malignas. Los cuidados paliativos requieren una intervención en el cuidado de los pacientes en etapas tempranas, avanzadas o terminales, estimulando un proceso de alivio o control de los signos y síntomas. En este contexto, la función del fisioterapeuta es actuar de forma complementaria al tratamiento paliativo, con el objetivo de mejorar la recuperación del individuo, restableciendo las funciones de diferentes partes del cuerpo. El objetivo de este trabajo es discutir la importancia de la intervención de fisioterapia en oncología, con un enfoque en los cuidados paliativos. Se refiere a una revisión de literatura narrativa. Para este artículo, se realizaron búsquedas manuales en la plataforma Google Scholar y artículos seleccionados relacionados con el tema. A partir de los datos presentados, el papel de la intervención fisioterapéutica se relaciona con la rehabilitación en todas las etapas de la enfermedad, en la que el dolor es la ocurrencia más relevante provocando un gran tormento al paciente. La fisioterapia es un área importante en el tratamiento de pacientes terminales, rehabilitando la condición motora y, por tanto, insertando a este individuo en la reanudación de la independencia funcional. Finalmente, los estudios demuestran que la fisioterapia tiene un papel importante en los cuidados paliativos, sin embargo, las definiciones de cuidados a los pacientes paliativos son necesarias para ser difundidas entre los fisioterapeutas y la investigación es necesaria para una buena mejora professional.
\end{abstract}

Palabras clave: Fisioterapia; Cuidados paliativos; Neoplasias.

\section{Introdução}

O câncer é uma patologia com características de ampliação celular sem controle que desencadeia um acúmulo de células de origem cancerígenas recebendo o nome de neoplasia maligna. Sendo assim, com a ascensão dessa doença, maior é a 
carência de atuação de uma equipe de saúde para atuar na desordem neoplásica. Neste contexto, têm como algumas particularidades a possibilidade de invadir tecidos próximos e a migração dessas células de origem tumoral por via circulatória ou linfática para órgão distais, levando assim pacientes a cuidados paliativos ou a óbito (Muller, 2011).

Segundo a Organização Mundial da Saúde (OMS), a atenção a pacientes em fase terminal, desenvolve um tratamento de cuidados aos indivíduos sem necessidade ou recursos de cura, estimulando um processo de amenização ou de controlar os sinais e sintomas: físicos, psicológicos e espirituais. Com isso, promover uma qualidade de vida para esses pacientes e a família, por meio da prevenção e alívio do sofrimento (Arrais, 2014).

Quando o diagnóstico é dado de maneira tardia, o tratamento geralmente é direcionado para os cuidados paliativos, pois a patologia frequentemente está em estágio avançada, devido a esse fator o tratamento curativo não é mais uma possibilidade para o paciente (Menossi; Lima; Corrêa, 2008). Para que os cuidados paliativos possam ser iniciados sem que haja intercorrência é necessário a formação de uma equipe multiprofissional, que vai ser responsável pelo desenvolvimento e comprimento de todo o programa de cuidados tanto ao paciente quanto aos seus familiares (Bueno et al., 2007).

A prestação de assistência faz-se necessária em todas as etapas da doença (Costa et al, 2007). Nesse contexto, o papel da fisioterapia é atuar de forma complementar ao tratamento paliativo, com o objetivo de melhorar a recuperação do paciente, dentro do seu alcance profissional, por meio de procedimentos que agem sobre o organismo humano, a fim de uma mobilização ativa ou passiva, restaurando a função de diferentes partes do corpo (Nascimento Paixão; Nadai Dias, 2012). Sendo assim, a fisioterapia auxilia na qualidade de vida dos indivíduos com a doença inicial ou em crescimento desta, reabilitando o funcionamento do paciente, bem como instrui o cuidador a lidar com a ascensão rápida da enfermidade (Florentino, 2012).

Dessa maneira, identifica-se como objetivo desse trabalho demonstrar a importância da atuação do profissional fisioterapeuta na área de oncologia, com enfoque principalmente nos cuidados paliativos, como pontos específicos: Mostrar os métodos mais utilizados no controle da dor dos pacientes e mostrar as fases dos cuidados paliativos.

\section{Método}

Refere-se a uma revisão narrativa de literatura, cuja finalidade é permitir que o resumo possa atualizar e obter informações acerca de um determinado tema em curto período de tempo. Assim, consiste em uma publicação que contempla o “estado da arte" de temáticas (Rother, 2007). Para este artigo foram realizadas buscas manuais na plataforma Google Scholar e a partir de uma óptica fundamentalmente voltada em sua grande parte para materiais e trabalhos já publicados, buscou-se abordar de maneira objetiva e coesa um acervo significativo de artigos científicos, os quais se dão por essência deste trabalho.

\section{Resultados e Discussão}

Para a Organização Mundial de Saúde (OMS) a caracterização dos cuidados paliativos foi realizada em meados de 1990, com as principais vertentes voltadas para os cuidados ativos e integrais para os pacientes com patologias que não possui resposta terapêutica para os tratamentos curativos. Estes cuidados possui um objetivo central mais no controle da dor e dos sintomas psicológicos, físicos, espirituais e sociais, visando a melhora da qualidade de vida dos pacientes em fase terminais e a de seus familiares (Silva; Sudigursky, 2008).

Segundo o autor Florentino (2012), a fisioterapia desempenha um papel muito importante nos cuidados paliativos, pois visa proporcionar um melhor tratamento aos pacientes com doenças avançadas ou progressivas, restaurando a função e a independência. Desta forma, os recursos preventivos também são um aspecto que sempre deve ser considerado por meio da fisioterapia. Nessa análise, Todos os profissionais envolvidos são responsáveis por antecipar possíveis complicações e tomar as devidas precauções necessárias para evitar sofrimentos desnecessários a pacientes e familiares. 
A Classificação Nacional Australiana de Pacientes Subagudos e Não Agudos (AN-SNAP) descreve quatro estágios de cuidados paliativos, dependendo da condição clínica do paciente e do estágio patológico. Esses estágios correspondem a vários graus de complexidade: há um estágio agudo Este é o desenvolvimento inesperado do problema ou o agravamento do problema existente; a exacerbação é definida pelo desenvolvimento gradual do problema, e o controle da situação não mudou repentinamente; a fase terminal, a morte do paciente é iminente; o último é o estábulo estágio, qualquer estágio anterior onde estão os pacientes que não estão incluídos (Girão, 2013).

Para o autor Taborelli (2010) a forma usada para o paciente para controlar a dor mesmo sem a possibilidade de se chegar à cura é uma das mais importantes formas de atuação do fisioterapeuta na área de cuidados paliativos, onde ele utiliza de métodos e recursos não invasivos, que irão ser úteis no controle da dor, disponibilizando com o que o paciente consiga o máximo de conforto possível.

Os problemas mais comuns encontrados nos pacientes oncológicos acabam por ter vários fatores e que podem ser complicados pela evolução da patologia subjacente e seu tratamento. Um dos sintomas mais recorrentes de pacientes oncológicos é a fraqueza geral, na qual pode ser resultante de inúmeros fatores, incluindo os efeitos mecânicos causados pelo tumor primário ou suas metástases no sistema nervoso central ou periférico, assim como, efeitos provenientes da radioterapia, quimioterapia, síndrome do imobilismo e disfunções de caráter psicológico entre outros (Melo, 2013).

\section{Terapia para a dor}

O mecanismo de análise da dor é iniciado com o diagnóstico clínico e deve ser acompanhando e observado por uma equipe de profissionais de várias áreas, visto que, o indivíduo com incômodo doloroso é decorrente de condições neurofisiológicas, emocionais, e sua percepção individual da dor, consequentemente, a equipe multiprofissional deve considerar as dores de forma abrangente. A dor geralmente é um sintoma bastante comum em uma neoplasia maligna, se trata de uma análise sensitiva e emocional, sendo um sentimento desconfortável, que pode ser pertencente a uma lesão real ou um agravamento dos tecidos, com isso, a associação internacional para estudo da dor considera ainda que cada paciente classifica o grau da dor e sua intensidade por meio de suas vivências individuais (Pena et al., 2008). Dessa maneira, com o sofrimento decorrente do impacto negativo da dor que causa uma piora nas condições para o bem-estar do paciente com câncer e em cuidados paliativos. Por consequência, é necessário técnicas eficazes para diminuir sensações pertinentes à dor que deverá ter a finalidade de redução desses sintomas (Inca, 2011).

A terapia de alívio de dor é algo de suma relevância nos cuidados paliativos, visto que busca a qualidade de vida do paciente. Nessa análise, a atenção fisioterapêutica utilizada é a eletroterapia por meio da eletroestimulação nervosa transcutânea (TENS), para a finalidade de monitoramento da dor aguda e crônica, mediante a diferentes mecanismos do TENS convencional e acupuntura. (Villanova et al. 2013).

\section{Cinesioterapia}

A cinesioterapia é recurso fisioterapêutico que é essencial no processo de reabilitação do paciente, na qual pode ter início com movimentos de forma passiva ou ativa e atividades com a finalidade de ascensão da musculatura. A técnica da cinesioterapia nesse meio se torna um aliado importante no tratamento, pois ajuda na evolução do ganho do desenvolvimento funcional na parte onde teve agravamentos, melhorando o movimento, a força, o desenvolvimento e a nutrição dos tecidos, a fim de evitar que o indivíduo entre em uma acinesia (Mozzini et al., 2007). Em relação ao contexto, essa técnica é de grande valia, visto que, utiliza movimentos de forma natural proporcionando uma coordenação motora, resistência à fadiga e um fortalecimento da força muscular (Sampaio et al., 2005). 


\section{Alívios dos sintomas psicofísicos}

A saúde mental de pacientes que convivem com uma enfermidade que não possui um tratamento terapêutico de cura viável, por consequência, acaba desenvolvendo estresse e a depressão intensificando a piora de uma estabilidade do seu quadro de saúde gerando distúrbios como: aumento do tensão musculoesquelético, caibras, hipertensão arterial, tremores e dores em diversas parte do corpo, com isso, as reações psicológicas e psiquiátricas incluem a deterioração dos distúrbios comportamentais ou problemas sociais, como aumento da ansiedade, irritabilidade, fraqueza, nervosismo, medo. (Bernik, 2006).

Nesse sentido, os recursos manuais aplicados na prática da fisioterapia auxiliam no relaxamento do organismo, como o yoga que é uma prática que trabalha o corpo e a mente, outras técnicas são: Watsu, que é um procedimento realizado em uma piscina aquecida; Tai chi, procedimento que é mediante a atividade física e relaxamento do corpo; Meditação, para autoconhecimento e redução do estresse. Esses procedimentos têm a finalidade de diminuir os sintomas decorrentes da doença para melhorar o bem-estar do paciente (Reiriz, 2009).

\section{Fisioterapia no pré-operatório e pós-operatório}

A intervenção cirúrgica é realizada por meio da remoção do tumor na região onde está sendo afetada e extração de órgãos endócrinos para dificultar ou bloquear a sua distribuição pelo organismo (Volpato et al., 2007). Nessa percepção, o papel do fisioterapeuta no período do pré-operatório consiste em levar informações dos recursos, técnicas e procedimentos fisioterapêuticos no pós-operatório após a cirurgia, e de forma tardia, além de ter uma visão mais abrangente do acometimento clínico e observar problemas que venham a interferir na reabilitação.

No que tange ao pós-operatório de início pós cirurgia, é fundamental a realização de uma nova análise do paciente seguindo os protocolos adequados e cuidados com o manejo do indivíduo e posteriormente o encaminhamento ao tratamento onco-funcional. Porém, quando essa avaliação é feita de forma tardia o fisioterapeuta deve analisar o caso clínico com a finalidade de meios para metas em um curto período e iniciando imediatamente o tratamento (Leite et al., 2013).

\section{Considerações Finais}

A fisioterapia vem ganhando seu espaço dentre as áreas médicas e assim mostrando sua grande importância, principalmente nas recuperações e tratamento de pacientes, foi possível verificar que o profissional fisioterapeuta tem um papel fundamental no auxílio da dor em pacientes oncológicos principalmente os que necessitam de cuidados paliativos pois, mesmo que não haja esperança de cura, possa amenizar os sintomas da patologia para tornar a vida do paciente mais estável possível que é o esperado tanto pelo indivíduo quanto pela família do mesmo, sendo assim, as definições de atenção a pacientes paliativos são serem necessárias propagadas entre os fisioterapeutas e pesquisas são necessárias para um bom aperfeiçoamento profissional.

Em virtude disso, deve-se ressaltar que, em relação aos privilégios de que gozam os autores das referências selecionadas nesta revisão de literatura, a escolha de teorias e métodos para realizar inovações pode ser considerada uma abordagem promissora para o desenvolvimento do conhecimento atual. Neste tópico, portanto, recomenda-se o uso de métodos qualitativos em pesquisas futuras e a adoção de teorias mais profundas que possam refletir sobre a experiência emocional e funcional dessa população antes, durante e após as complicações oncológicas que levam aos cuidados paliativos.

\section{Referências}

Arrais, R. C. S. (2014). Atuação da fisioterapia nos cuidados paliativos oncológicos. Unicamp. http://docplayer.com.br/6410478-Atuacao-da-fisioterapia-noscuidados-paliativos-oncologicos.html 
Research, Society and Development, v. 10, n. 6, e46310616042, 2021

(CC BY 4.0) | ISSN 2525-3409 | DOI: http://dx.doi.org/10.33448/rsd-v10i6.16042

Bueno, M. et al. (2007). Reflexões sobre cuidados paliativos no período neonatal. Prática hospitalar., São Paulo, 9(50), 87-90.

Bernik, V. (2006). Estresse: o assassino silencioso. https://www. cerebromente.org.br/n03/doencas/stress.htm.

Costa, C. A. et al. (2007). Dor oncológica. Revista portuguesa de pneumologia, 13(6), 855-867.

Florentino D. M. et al. (2012). A fisioterapia no alívio da dor: uma visão reabilitadora em cuidados paliativos. Revista Hospital Universitário Pedro Ernesto, $11(2), 50-57$.

Girão, M., et al. (2013). Fisioterapia nos cuidados paliativos. Revista de Ciências da Saúde da ESSCVP 5(1), 1-8.

Inca. (2011). Informativo sobre o câncer. https://www.inca.gov.br/publicacoes/informativos

Leite, F. et al. (2013) Rotinas de atividades desempenhadas em um programa de reabilitação onco-funcional: um relato de experiência. Congresso Brasileiro de Fisioterapia em oncologia, 14(4), 22-28.

Melo, T. P. T., et al. (2013). A Percepção dos Pacientes Portadores de Neoplasia Pulmonar Avançada diante dos Cuidados Paliativos da Fisioterapia. Revista Brasileira de Cancerologia, 59(4), 547-553.

Mozzini, C. B.; Mozzini, A.; Suchuster, R. C. (2017). O esvaziamento cervical e o papel da fisioterapia na sua reabilitação. Revista Brasileira de Cancerologia, 53(1), 55-61.

Muller, A. M., et al. (2011) Paciente Oncológico em Fase Terminal: Percepção e Abordagem do Fisioterapeuta. Revista Brasileira de Cancerologia, 57(2), 207-215.

Nascimento, P. R. C.; Nadal, D. L. I. (2012) A atuação da fisioterapia nos cuidados paliativos da criança com câncer. Ensaios e Ciência, $16(4), 153-169$.

Pena, R. Barbosa, L. A.; Ishikawa, N. M. (2008) Estimulação transcutânea do nervo (TENS) na dor oncológica: uma revisão de literatura. Revista brasileira cancerologia, 54(2), 193-199.

Reiriz, A. B. et al. (2009). A inclusão da fisioterapia nos cuidados paliativos. Prática Hospitalar, 11(66), 113-115.

Rother, E. T. (2017). Revisão sistemática X revisão narrativa. Acta Paulista de Enfermagem, 20(2), 5-6.

Menossi, J. M.; Lima, R. A. G.; Corrêa, A. K. (2008). Pain and the challenge of interdisciplinarity in child care. Revista Latino-Americana de Enfermagem., Ribeirão Preto, 16(3) 489-94.

Sampaio, L. R.; Moura, C. V.; Resende, M. A. (2005). Recursos fisioterapêuticos no controle da dor oncológica: revisão de literatura. Revista brasileira cancerologia, 51(4), 339-346.

Silva, E. P.; Sudigursky, D. (2008). Conceptions about palliative care: literature review. Acta Paulista de Enfermagem, São Paulo, $21(3), 504-508$.

Tamborelli. V., et al. (2010). O papel da enfermagem e da fisioterapia na dor em pacientes geriátricos terminais. Revista brasileira de Geriatria \& Gerontologia, 4(3), 146- 153.

Villanova, V. H. Fornazari, L. P.; Deon, K. C. (2013). Estimulação elétrica nervosa transcutânea como coadjuvante no manejo da dor oncológica. Revista Inspirar Movimento \& Saúde, 6(5), 28-33.

Volpato, L. E. R.; et al. (2007) Mucosite bucal rádio e quimio induzida. Revista Brasileira Otorrinolaringol, 73(4), $562-568$. 